\title{
A CHARACTERIZATION OF NONATOMIC HILBERT ALGEBRAS
}

\author{
ALESSANDRO FIGÀ-TALAMANCA AND GIANCARLO MAUCERI
}

\begin{abstract}
We say that a Hilbert algebra is atomic if its fulfillment is generated by its minimal projections. We prove that Hilbert algebra $\mathcal{Q}$ is not atomic if and only if there is an infinite group $\mathcal{G}$ of unitary elements of the von Neumann algebra generated by $\mathcal{Q}$, and an element $\xi_{0}$ of the fulfillment of $Q$, which commutes with every element of $\mathcal{G}$, and such that set $\left\{U \xi_{0}\right.$ : $U \in \mathcal{G}\}$ is orthonormal. This result is then applied to gain information on the Plancherel measure of certain unimodular groups.
\end{abstract}

Introduction and statement of the results. Let $\mathcal{Q}$ be a Hilbert algebra. We will make use of the basic results on Hilbert algebras contained in [5], and we will adopt the terminology (but not necessarily the notation) used there. Let $\mathcal{H}$ be the Hilbert space completion of $\mathcal{Q}$. If $a \in \mathbb{Q}$, we let $\lambda(a)$ (respectively $\rho(a)$ ) be the bounded operator on $\mathcal{H}$ obtained by extending by continuity the map $b \rightarrow a b\left(b \rightarrow b a\right.$, respectively) to all of $\mathcal{H}$. Then $\lambda$ is a ${ }^{*}$-isomorphism, and $\rho$ a ${ }^{*}$-anti-isomorphism of $\mathcal{Q}$ into the algebra $\mathcal{L}(\mathcal{H})$ of all bounded linear operators on $\mathcal{H}$. We let $\mathcal{W}$ be the von Neumann algebra generated by $\{\lambda(a)$ : $a \in \mathbb{Q}\}$ and $\mathcal{W}^{\prime}$ the von Neumann algebra generated by $\{\rho(a): a \in \mathbb{Q}\}$. The algebra $\mho^{\prime}$ is then the commutant of $\mathscr{W}$. Let $\xi \rightarrow \xi^{*}$ be the unique extension to $\mathcal{H}$ of the involution on $\mathcal{Q}$. An element $\xi \in \mathcal{H}$ is called bounded if the map $a \rightarrow \rho(a) \xi$ of $\mathcal{Q}$ into $\mathcal{H}$ is continuous in the Hilbert space norm. If $\xi$ is bounded then the map $a \rightarrow \lambda(a) \xi$ is also continuous. The set of bounded elements of $\mathcal{H}$ is canonically a Hilbert algebra which contains $\mathbb{Q}$ and is called the fulfillment of $\mathcal{Q}$. We denote by $\overline{\mathcal{Q}}$ the fulfillment of $\mathbb{Q}$ and we call a Hilbert algebra full if $\mathcal{Q}=\overline{\mathcal{Q}}$. For $\xi \in \overline{\mathcal{Q}}$ we let $\lambda(\xi)$ and $\rho(\xi)$ be the elements of $\mathscr{W}$ and $\mathscr{W}^{\prime}$, respectively, whose restriction to $\mathcal{Q}$ is defined as follows:

$$
\lambda(\xi) a=\rho(a) \xi ; \quad \rho(\xi) a=\lambda(a) \xi .
$$

For $\xi \in \overline{\mathcal{Q}}$, define $\phi\left(\lambda(\xi)^{*} \lambda(\xi)\right)=\langle\xi, \xi\rangle$. Then $\phi$ extends to a faithful normal semifinite trace defined on the positive elements of $\mathscr{W}$. We will also use the same symbol $\phi$ for the linear extension of $\phi$ to the space of linear combinations of positive elements of $\mho$ with finite trace. We shall reserve the symbol $\operatorname{Tr}$ to denote the ordinary trace of positive operators on a Hilbert space.

DEFINITION. We call a Hilbert algebra $\mathbb{Q}$ atomic if its fulfillment $\overline{\mathcal{Q}}$ is generated by minimal projections. We say that $\mathbb{Q}$ is nonatomic if it is not atomic.

Received by the editors October 14, 1976 and, in revised form, January 19, 1978.

AMS (MOS) subject classifications (1970). Primary 46L10; Secondary 43A70. 
We shall prove the following:

THEOREM. $\mathcal{Q}$ is nonatomic if and only if there exists an infinite group $\mathcal{G}$ of unitary operators of $\mathscr{W}$, and a bounded element $\xi_{0} \in \overline{\mathcal{Q}}$, such that $\lambda\left(\xi_{0}\right) U=$ $U \lambda\left(\xi_{0}\right)$ for $U \in \mathcal{G}$, and the set $\left\{U \xi_{0}: U \in \mathcal{G}\right\}$ is orthonormal in $\mathcal{H}$.

The result just stated was motivated by the study of unimodular groups with atomic Plancherel measure, that is groups for which the Hilbert algebra $\bigodot_{00}(G)$ of the continuous functions with compact support is atomic. When $G$ is noncompact these are the so-called Fell groups, studied in [4]. A consequence of our theorem is the following

COROLlary. If $G$ is a unimodular group and the Plancherel measure on the space of irreducible representations $\hat{G}$ is purely atomic, then there is no compact neighborhood $K$ of the identity in $G$ which is invariant with respect to an infinite discrete subgroup $D$, and such that $K \cap D=\{1\}$.

One implication of our theorem can be formulated in a stronger fashion (as the proof clearly shows): if $\mathcal{Q}$ is nonatomic then its fulfillment contains a Hilbert subalgebra isomorphic to the full Hilbert algebra of the discrete commutative group which is the weak direct product of infinitely many copies of the group of two elements. This implication of the Theorem, for the case of $\bigodot_{00}(G)$, was already implicit in the construction of "Walsh operators" in [2, p. 40]. Finally we want to point out the similarity between the condition on $\mathcal{G}$ given in our theorem and the condition of nondegeneracy for a commutative group of unitary operators, considered by W. Arveson in [1]. Similar conditions for noncommutative groups are studied in [3].

Proof of the Theorem. Then conditions whose equivalence is asserted in the Theorem concern the fulfillment of $Q$. Therefore we may as well assume that $\mathbb{Q}=\overline{\mathbb{Q}}$ is a full Hilbert algebra. Let $\mathcal{E}$ be the set of selfadjoint idempotents of $Q$. If $e_{1}, e_{2} \in \mathcal{E}$ we say that $e_{1}$ contains $e_{2}$ if there is $e_{3} \in \mathcal{E}$ such that $e_{1}=e_{2}+e_{3}$ and $e_{2} e_{3}=e_{3} e_{2}=0$. Suppose now that $\mathcal{Q}$ is nonatomic. Then there exists an element $e \in \mathcal{E}$ which contains no minimal idempotent. We may assume, without loss of generality, that $\phi(\lambda(e))=\langle e, e\rangle=1$. Since $e$ contains no minimal idempotent, for every $0<t<1$, there exists $e_{t}$ contained in $e$ such that $\left\langle e_{t}, e_{t}\right\rangle=t[2, \mathrm{p} .40]$. Thus proceeding by induction we can construct, for every positive integer $n$, commuting idempotents $e_{n 1}, \ldots, e_{n 2} n$, such that $\left\langle e_{n j}, e_{n j}\right\rangle=2^{-n}, e_{n 1}+\cdots+e_{n 2} n=e$, and $e_{n, 2 j-1}+$ $e_{n, 2 j}=e_{n-1, j}$ (For details of this construction cf. [2, p. 40].) Let

$$
R_{n}=\sum_{j=1}^{2^{n}}(-1)^{j+1} \lambda\left(e_{n j}\right)+I-\lambda(e),
$$

where $I$ is the identity of $\mathscr{W}$. Then $R_{n}^{*}=R_{n}$ and $R_{n}^{2}=I$. Thus $R_{n}$ is a unitary operator of $\mho$. Let $\mathcal{G}$ be the group generated by the family $\left\{R_{n}\right\}$. If $U=R_{j_{1}} \cdots R_{j_{s}}, j_{1}<j_{2}<\cdots<j_{s}$, is an element of this group, other than 
the identity, then

$$
\phi(\lambda(e) U)=\langle U e, e\rangle=\prod_{k=1}^{s}\left\langle R_{j_{k}} e, e\right\rangle
$$

(this because of the independence of the operators $R_{j_{k}}$ with respect to the trace); but for each $R_{n}$,

$$
\phi\left(\lambda(e) R_{n}\right)=\left\langle R_{n} e, e\right\rangle=\frac{1}{2^{n}} \sum_{j=1}^{2^{n}}(-1)^{j+1}=0 .
$$

Therefore $\phi(\lambda(e) U)=0$. Let $U_{1}, U_{2} \in \mathcal{G}$ and $U_{1} \neq U_{2}$, then $\left\langle U_{1} e, U_{2} e\right\rangle=\left\langle U_{2} U_{1} e, e\right\rangle=0$ because $U_{2} U_{1} \neq 1$. Moreover $\lambda(e) R_{n}=R_{n} \lambda(e)$ for each $n$, therefore $\lambda(e) U=U \lambda(e)$ for every $U \in \mathcal{G}$. Letting $\xi_{0}=e$ we obtain the condition of the Theorem. Conversely suppose that there exist $\xi_{0}$ and $\mathcal{G}$ as in the statement of the Theorem. We shall prove first of all that $\mathscr{S}$ contains a von Neumann subalgebra isomorphic to the von Neumann algebra $\mathrm{VN}(\mathcal{G})$ generated by the left action of $\mathcal{G}$ on $l^{2}(\mathcal{G})$. We notice that the subspace $\mathcal{K}_{0}$ of $\mathcal{H}$, generated by $\left\{U \xi_{0}: U \in \mathcal{G}\right\}$ is isometric to $l^{2}(\mathcal{G})$, under the correspondence $\Phi$ which associates $U \xi_{0}$ to the function on $\mathcal{G}$ which is one at $U$ and zero elsewhere. Let $\mathcal{W}_{0}$ be the von Neumann subalgebra of $\mathscr{W}$ generated by $\mathcal{G}$. Then the map $T \rightarrow \Phi T \Phi^{-1}$, maps $\mathcal{W}_{0}$ isometrically onto $\mathrm{VN}(\mathcal{G})$. It is not difficult to see that this map is also weak* continuous, indeed if $f_{j}, g_{j} \in l^{2}(\mathcal{S}), \Sigma\left\|f_{j}\right\|_{l^{2}}\left\|g_{j}\right\|_{l^{2}}<\infty$, and $T \in \mho_{0}$ then

$$
\sum\left\langle\Phi T \Phi^{-1} f_{j}, g_{j}\right\rangle=\Sigma\left\langle T \Phi^{-1} f_{j}, \Phi^{-1} g_{j}\right\rangle \text {. }
$$

Thus $\left\{T:\left|\Sigma\left\langle\Phi T \Phi^{-1} f_{j}, g_{j}\right\rangle\right|<\varepsilon\right\}$ is a weak* open set in $\mho_{0}$, and $T \rightarrow$ $\Phi T \Phi^{-1}$ is weak* continuous. Let $\omega_{0}(T)=\left\langle T \xi_{0}, \xi_{0}\right\rangle$ for $T \in \mho_{0}$. Then $\omega_{0}$ is a weak* continuous positive linear functional with the property that $\omega_{0}(T)=$ $\psi(T)$, where $\psi$ is the canonical finite faithful normal trace on the algebra $\mathrm{VN}(\mathcal{G})$. (Elements of $\mathrm{VN}(\mathcal{G})$ may be identified with functions on $\mathcal{G}$, and $\psi$ is just the evaluation at the identity of $\mathcal{G}$.) Now $\mathrm{VN}(\mathcal{G})$ contains no minimal projections [2, \$4]. Therefore we may find as in [2, p. 40] for each $n$, a partition of the identity $\left\{P_{n 1}, \ldots, P_{n 2^{n}}\right\}$; consisting of projections $P_{n j} \in$ $\mathrm{VN}(G)$, such that:

$$
\begin{array}{cc}
P_{n j} P_{m k}=P_{m k} P_{n j}, & P_{n 1}+\cdots+P_{n 2^{n}}=I, \\
\psi\left(P_{n j}\right)=2^{-n}, & P_{n-1, j}=P_{n, 2 j-1}+P_{n, 2 j} .
\end{array}
$$

Let $P_{n j}^{\prime}=\Phi^{-1} P_{n j} \Phi$. Then $\left\{P_{n 1}^{\prime}, \ldots, P_{n 2^{n}}^{\prime}\right\}$ is a partition of the identity in $\mho$, consisting of projections of $\mathscr{W}_{0}$, and $\omega_{0}\left(P_{n j}^{\prime}\right)=2^{-n}$. We notice that every element of $\mathscr{U}_{0}$ commutes with $\lambda\left(\xi_{0}\right)$ and therefore $P_{n j}^{\prime} \lambda\left(\xi_{0}\right)=\lambda\left(\xi_{0}\right) P_{n j}^{\prime}$.

We shall now draw a contradiction from the assumption that $\mathbb{Q}$ is atomic. We recall a few facts easily deducible from the contents of $\$ \S 5$ and 6 of [5] concerning full atomic Hilbert algebras. If $\mathcal{S}$ is a maximal set of unequivalent irreducible subrepresentations of $\lambda$ (i.e. square integrable representations) then, for each $\sigma \in \mathcal{S}$, there is a unique minimal *-closed two sided ideal $\mathbb{C}_{\sigma}$ 
on which $\sigma$ is faithful. The ideal $\mathbb{Q}_{\sigma}$ is complete as a Hilbert space and it depends only on the equivalence class of $\sigma$. Furthermore $\mathcal{H}=\bigoplus_{\sigma \in \delta} \mathbb{Q}_{\sigma}$. If $\sigma$, $\sigma^{\prime} \in \mathcal{S}$ and $\sigma \neq \sigma^{\prime}$, then $\sigma^{\prime}\left(\mathbb{Q}_{\sigma}\right)=0$. We also have that $\sigma$ maps $\mathbb{Q}$ and hence $\mathbb{Q}_{\sigma}$ onto the algebra of all Hilbert-Schmidt operators on the space $\mathcal{H}_{\sigma}$ on which $\sigma$ acts. If $a, b \in \mathbb{Q}$ then

$$
\langle a, b\rangle=\sum_{\sigma \in \delta} d_{\sigma} \operatorname{Tr}\left(\sigma\left(b^{*}\right) \sigma(a)\right)
$$

where $d_{\sigma}$ is a positive number which equals the square of the norm of any minimal idempotent in $\mathcal{Q}_{\sigma}$. Let $T \in \mathcal{W}$ then $\sigma(T a)=\sigma(T) \sigma(a)$, where $\sigma(T)$ $\in \mathcal{L}\left(\mathcal{H}_{\sigma}\right)$. The correspondence $T \rightarrow \sigma(T)$ maps $\mathscr{W}$ onto $\mathcal{L}\left(\mathcal{H}_{\sigma}\right)$ and $\sigma(\lambda(a))$ $=\sigma(a)$. We fix now $\bar{\sigma} \in \mathcal{S}$ such that $\bar{\sigma}\left(\xi_{0}\right) \neq 0$. Let $T_{0}=\bar{\sigma}\left(\xi_{0}^{*} \xi_{0}\right)$. Then $T_{0} \in \mathcal{E}\left(\mathcal{H}_{\sigma}\right)$ is a nonzero operator of trace class, and

$$
T_{0} \bar{\sigma}\left(P_{n j}^{\prime}\right)=\bar{\sigma}\left(\lambda\left(\xi_{0}^{*} \xi_{0}\right) P_{n j}^{\prime}\right)=\bar{\sigma}\left(P_{n j}^{\prime} \lambda\left(\xi_{0}^{*} \xi_{0}\right)\right)=\bar{\sigma}\left(P_{n j}^{\prime}\right) T_{0}
$$

for every $n$ and $j$. Let $Q \in \mathcal{L}\left(\mathcal{H}_{\bar{\sigma}}\right)$ be a spectral projection of $T$ such that $T Q=\alpha Q$ with $\alpha>0$. Such a projection exists because $T$ is a compact positive operator. Choose $n$ so that $2^{-n}<d_{\bar{\sigma}} \alpha$. Since $\bar{\sigma}\left(P_{n 1}^{\prime}\right)+\cdots+$ $\bar{\sigma}\left(P_{n 2^{1}}^{\prime}\right)$ is the identity in $\mathcal{L}\left(\mathcal{H}_{\bar{\sigma}}\right)$, we have that $Q \bar{\sigma}\left(P_{n 1}^{\prime}\right)+\cdots+Q \bar{\sigma}\left(P_{n 2^{n}}^{\prime}\right)=$ $Q$. But $Q$ is a spectral projection of $T_{0}$, therefore it commutes with all the operators which commute with $T_{0}$, in particular $Q \bar{\sigma}\left(P_{n j}\right)=\bar{\sigma}\left(P_{n j}\right) Q$ and for each $j, Q \bar{\sigma}\left(P_{n j}\right)$ is a projection. Now for some $j, Q \bar{\sigma}\left(P_{n j}\right) \neq 0$, therefore $\operatorname{Tr}\left(Q \bar{\sigma}\left(P_{n j}\right)\right) \geqslant 1$. On the other hand,

$$
\begin{aligned}
2^{-n} & =\omega_{0}\left(P_{n j}^{\prime}\right)=\left\langle P_{n j}^{\prime} \xi_{0}, \xi_{0}\right\rangle \\
& =\sum_{\sigma \in \mathcal{\delta}} d_{\sigma} \operatorname{Tr}\left(\sigma\left(P_{n j}^{\prime}\right) \sigma\left(\xi_{0}^{*}\right) \sigma\left(\xi_{0}\right)\right) \geqslant d_{\bar{\sigma}} \operatorname{Tr}\left(T_{0} \bar{\sigma}\left(P_{n j}^{\prime}\right)\right) \\
& =\alpha d_{\bar{\sigma}} \operatorname{Tr}\left(Q \bar{\sigma}\left(P_{n j}^{\prime}\right)\right)+d_{\bar{\sigma}}\left(T_{0}(I-Q) \bar{\sigma}\left(P_{n j}^{\prime}\right)\right) \geqslant \alpha d_{\bar{\sigma}}>2^{-n} .
\end{aligned}
$$

This is the required contradiction and the Theorem is proved.

Proof of the Corollary. Suppose the conclusion does not hold. Let $C_{00}(G)$ be the Hilbert algebra of the statement of the Theorem, and $\xi_{0}$ be the characteristic function of $K$. Let $\left\{L_{x}: x \in D\right\}$ be the group of unitary operators of the Theorem, where $L_{x}$ indicates left translation by $x$ : for $\xi \in C_{00}(G), L_{x} \xi(y)=\xi\left(x^{-1} y\right)$. Then the condition of the Theorem is satisfied and we conclude that $\bigodot_{00}(G)$ is nonatomic.

\section{REFERENCES}

1. W. Arveson, A theorem on the action of abelian unitary groups, Pacific J. Math. 16 (1966), 205-212.

2. C. Cecchini and A. Figà-Talamanca, Projections of uniqueness for $L^{p}(G)$, Pacific J. Math. 51 (1974), 37-47.

3. A. Figà-Talamanca, On the action of unitary groups on a Hilbert space (Sympos. Harmonic Analysis and Spaces of Functions on Locally Compact Groups, Istituto Nazionale di Alta 
Matematica, Roma, 1976), Symposia Mathematica (to appear).

4. G. Mauceri, Square-integrable representations and the Fourier algebra of a unimodular group, Pacific J. Math. 73 (1977), 143-154.

5. M. Rieffel, Square-integrable representations of Hilbert algebras, J. Functional Analysis 3 (1969), 265-300.

Istituto Matematico, Universitì di Roma, Cittì Universitaria, 00100 Roma, Italy 